\title{
LE SKI... UN SPORT «ALPIN » ET DIRIGÉ PAR DES SUISSES ?
}

\section{Sébastien Cala et Grégory Quin}

De Boeck Supérieur | «Staps »

2019/3 n 125 | pages 89 à 105

ISSN 0247-106X

ISBN 9782807393394

Article disponible en ligne à l'adresse :

https://www.cairn.info/revue-staps-2019-3-page-89.htm

Distribution électronique Cairn.info pour De Boeck Supérieur.

(C) De Boeck Supérieur. Tous droits réservés pour tous pays.

La reproduction ou représentation de cet article, notamment par photocopie, n'est autorisée que dans les limites des conditions générales d'utilisation du site ou, le cas échéant, des conditions générales de la licence souscrite par votre établissement. Toute autre reproduction ou représentation, en tout ou partie, sous quelque forme et de quelque manière que ce soit, est interdite sauf accord préalable et écrit de l'éditeur, en dehors des cas prévus par la législation en vigueur en France. Il est précisé que son stockage dans une base de données est également interdit. 


\title{
Le ski... un sport « alpin » et dirigé par des Suisses ? \\ Skiing. .. an "alpine" sport ruled by Swiss leaders?
}

\author{
Sébastien CALA \\ Institut des Sciences du Sport de l'Université de Lausanne \\ Université de Lausanne \\ Quartier Unicentre \\ Bâtiment Synathlon \\ CH- 1015 Lausanne \\ sebastien.cala@unil.ch \\ Grégory QUIN \\ Institut des Sciences du Sport de l'Université de Lausanne \\ Université de Lausanne \\ Quartier Unicentre \\ Bâtiment Synathlon \\ CH- 1015 Lausanne \\ gregory.quin@unil.ch
}

Résumé : La figure de Marc Hodler plane sur la Fédération Internationale de Ski et sur le ski international. Par son mandat de 47 ans à la tête de l'institution (1951-1998), le dirigeant bernois est incontestablement le dirigeant sportif resté le plus longtemps dans une position majeure au sein du sport international, mais loin d'être celle un homme isolé, cette trajectoire incarne la densité des réseaux du ski suisse, rassemblant des acteurs du tourisme sportif, des figures de championnes et de champions, des organisateurs de compétitions et des politiques actifs aux différents niveaux du système helvétique. Dans le cadre de cette contribution, notre ambition est double, à la fois de comprendre les logiques suisses et internationales de la construction d'une gouvernance du ski autour de la Deuxième Guerre mondiale, mais aussi, de manière plus qualitative, de mesurer l'influence des dirigeants helvétiques sur les transformations des différentes modalités de ski (alpin, nordique) à cette même période. De ce fait, il s'agira pour nous de souligner les dynamiques croisées qu'il peut exister entre les deux principales régions clés du ski en Suisse (la Lutschine et les Grisons), mais aussi alors d'approfondir notre compréhension de l'organisation des réseaux helvétiques autour du ski international. Pour produire ces analyses, nous nous appuierons sur les archives institutionnelles de la FIS, mais aussi sur des documents consultés auprès de la Fédération Suisse de Ski, du Musée du sport suisse, de la commune de St. Moritz et du Comité International Olympique.

Mots-cLÉs : Ski, Suisse, Dirigeant, Réseaux, Alpes

ABstracr: This article examines how sports leaders from the People's Republic of China (PRC) served as key figures in building and leading new transnational networks in the time between the establishment of the PRC in 1949 and the start of the Cultural Revolution in 1966-even as constantly changing political tides, both domestically and internationally, affected their ability to do so. It begins with a brief discussion of the new sports leadership in the early 1950s before then tracing China's involvement in the socialist bloc sports networks and competitions, as well as its attempts to join and participate in the Olympics. The article then moves on to discuss how Chinese sports leaders in the 1960s, in particular Rong Gaotang and Huang Zhong, helped orchestrate the Games of the New Emerging Forces (GANEFO) - a politically motivated sporting federation 
and competition established in the aftermath of the Sino-Soviet split to assert Chinese socialism in the world, while also challenging the IOC's domination of international sport. Next, the article looks at the turn of events in late 1966, during the early Cultural Revolution, when State Sports Commission leader Rong Gaotang-involved in most diplomatic and high-level political activities-was denounced and criticized for his past activities. Just before the first (and last) Asian GANEFO was held in December 1966, the Chinese leadership in charge suddenly replaced Rong as China's delegation leader, and China soon retreated from the world of international sport for several years. The conclusion section notes how Rong's rehabilitation in early 1979, after Mao's death, the end of the Cultural Revolution, and the rise of Deng Xiaoping, was no coincidence: it came just as the new PRC leadership decided to return to the IOC as part of the plan to position China on the world stage as "open," "reformed," and "modernized."

KEYWORDs: China, international sport, diplomacy, competitions

Au lendemain de la Première Guerre mondiale, le ski international n'est pas encore structuré. En effet, il n'existe aucun organe international référent, même si des réunions entre représentants d'associations nationales ont déjà eu lieu sous l'impulsion des pays scandinaves ou en Europe centrale. Il faut attendre le premier grand événement international à Chamonix, en 1924, avec la "Semaine internationale des sports d'hiver » (Arnaud, 1991), pour que le monde du ski s'institutionnalise sous la forme d'une fédération internationale. Dans un premier temps, ce sont les Scandinaves qui occupent les postes clefs et ont une grande influence au sein de la Fédération internationale de ski (FIS). Ainsi, le premier président est suédois, Ivar Homquist, et son successeur, de 1934 à 1951, est le Norvégien Nikolai Ramm Östgaard. Cette prédominance des pays du Nord de l'Europe s'explique en grande partie par l'avancée technique des skieurs scandinaves sur leurs homologues européens ou nord-américains. Cette quasi-hégémonie n'est plus d'actualité au sortir de la Seconde Guerre mondiale avec l'élection du Suisse Marc Hodler à la présidence de la FIS en 1951. Le ski suisse prend alors une place de plus en plus importante sur la scène internationale, à la faveur du renversement des équilibres entre les différentes modalités de la pratique.

La trajectoire de Marc Hodler semble incarner à elle seule l'histoire du ski au $\mathrm{XX}^{\mathrm{e}}$ siècle. Après un mandat de 47 ans à la tête de la FIS, entre 1951 et 1998, le Bernois est incontestablement le dirigeant sportif resté le plus longtemps dans une position majeure au sein du sport international. Loin d'être un cas isolé, cette trajectoire incarne un "imaginaire » du ski suisse, rassemblant des images d'un tourisme sportif, de figures de championnes et de champions, des organisations de compétitions et surtout l'engagement de dirigeants jusqu'au plus haut niveau de l'administration de la discipline. De fait, Marc Hodler n'est pas une figure isolée, il représente une «tête de réseaux » que nous allons précisément chercher à décrire pour les décennies s'écoulant entre les années 1920 et la fin des années 1960.

Si la littérature concernant les "réseaux " (Lemercier, 2005), les « élites » ou autres « cartels » en Suisse est fournie (Humair, Guex, Mach \& Eichenberger, 2012 ; Mach, David, Ginalski \& Bühlmann, 2016), aucun historien ne s'est intéressé spécifiquement aux réseaux du sport suisse, et par conséquent jamais au cas du ski. Quelques travaux de recherches récents mettent en évidence les liens entre intérêts privés (essentiellement touristiques) et intérêts sportifs, que ce soit dans les grandes stations grisonnes (Seger, 2006), dans la région de Montreux (Guex, Roy \& Sauthier, 2012) ou dans le Jura suisse (Cala, 2019). Cependant, ces travaux mettent en évidence des dynamiques locales, voire régionales, sans jamais essayer de dégager des cadres "nationaux " ou de se concentrer sur les lieux de sociabilité que sont les organisations faîtières du ski 
en Suisse. Dans le même temps, les travaux de Laurent Tissot et Cédric Humair ont quant à eux abordé le sujet dans leurs travaux relatifs au développement du tourisme dans les Alpes, notamment sous l'angle du développement économique (Humair \& Tissot, 2011 ; Humair, Gigase, Lapointe Guigoz \& Sulmoni, 2014 ; Gigase, Humair \& Tissot, 2014), mais sans mobiliser prioritairement les archives du monde du ski. De la même manière, pour la France voisine, les travaux de Pierre-Olaf Schut (2013) ou d'Olivier Hoibian soulignent les réseaux d'acteurs existant autour de l'essor de l'alpinisme (Hoibian, 2006), mais ils ne se focalisent pas sur l'activité "ski alpin ». Pierre-Olaf Schut relève également, avec Éric Levet-Labry, l'importance des réseaux touristiques dans l'acquisition et l'organisation des Jeux olympiques de Chamonix en 1924 (Schut \& LevetLabry, 2014), ce que de premières recherches à Saint-Moritz confirment également pour 1928. Il faut toutefois relever que certains travaux apportent également des éléments intéressants à cette thématique, en dehors même de la prise en compte des ouvrages commémoratifs des principaux ski-clubs de Suisse'. Citons notamment ceux de Thomas Busset qui a étudié la diffusion du ski dans les territoires helvétiques ainsi que le développement d'une économie autour de la fabrique des skis (Busset, 2005, 2016) ou encore ceux de Peter Engel sur la médiatisation précoce du ski (Engel, 2013) ou plus récemment autour des candidatures suisses pour les Jeux olympiques, même si l'ouvrage se base sur une lecture « internaliste " des dynamiques des organisations sportives (Lacotte, Kiuri \& Stricker, 2017). Anne Philipona Romanens s'est pour sa part intéressée au rôle de l'armée dans la démocratisation du ski, notamment dans le canton de Fribourg (Philipona Romanens, 1999). Dans un texte consacré à l'Interassociation suisse pour le ski, Grégory Quin pose quant à lui des jalons autour des dynamiques qui préfigurent à la professionnalisation et à l'institutionnalisation de l'enseignement du ski en Suisse à partir de l'entre-deux-guerres (Quin, 2017), des jalons complétés dans une publication récente avec Sébastien Cala sur les déterminants de l'émergence des modalités « alpines » du ski (Quin \& Cala, 2019).

Dès lors, notre travail souhaite mettre en lumière les liens qui unissent les différents acteurs du ski au niveau suisse ainsi que les dynamiques suprarégionales qui permettent le développement de la discipline et de son économie touristique notamment.

Pour produire ces analyses, nous nous appuierons sur les archives institutionnelles de la FIS, de l'Interassociation suisse pour le ski (IASS), de l'Association des Écoles de ski suisses (AESS), mais aussi sur des documents consultés auprès de la Fédération suisse de ski (anciennement nommée Association suisse des clubs de ski (ASCS)), du Musée du sport suisse, du Comité national pour le sport d'élite (CNSE), du Comité international olympique (CIO) et de l'Office du tourisme (Kurund Verkehrsverein) de Saint-Moritz. Sur ces bases, pour développer notre argumentation, nous suivrons un plan chronologique, afin de nous pencher successivement sur l'entredeux-guerres, sur les années conduisant à la « débâcle de 1964 » et enfin sur les conséquences de cet échec. Dès lors, dans la première partie, notre ambition est de souligner comment les réseaux du ski suisse vont se constituer, dans l'entre-deux-guerres, autour de logiques diverses, mais dont les effets vont se renforcer. Ainsi, les dirigeants suisses du ski possèdent souvent des ancrages géographiques communs, siègent dans des institutions communes (qu'ils contribuent à mettre en place à cette période) et sont impliqués dans une promotion touristique qui va changer de dimension, à la fois sous l'influence d'un ralentissement économique et d'un essor technique autour des premières infrastructures

1 Une histoire de ces ouvrages serait d'ailleurs à produire, aussi en comparaison internationale, pour appréhender les logiques commémoratives et patrimoniales singulières qui se développent dans les milieux du ski. 
permettant la pratique du ski de descente. La deuxième partie sera consacrée à la période de 1951 - élection de Marc Hodler à la présidence de la FIS - à l'échec helvétique lors Jeux olympiques de 1964. Nous relèverons ici le renforcement des réseaux du ski suisse au sein des instances internationales mais également au niveau national à la fois sur la scène politique et industrielle. Enfin, nous reviendrons sur les conséquences des échecs helvétiques lors des Jeux olympiques d'Innsbruck (Quin, 2018) et analyserons l'importance des réseaux précités dans la refonte du système sportif suisse.

\section{La structuration des réseauX suisses AUTOUR DU SKI DURANT L'ENTRE-DEUX-GUERRES}

En préambule, il est important de présenter un bref rappel des débuts du ski en Suisse. Cela permet en effet de contextualiser le propos et permet surtout une meilleure compréhension de la situation et des structures du ski helvétique au sortir de la Première Guerre mondiale.

Le ski fait son apparition en Suisse à la fin du XIXe siècle et c'est en 1893 que le premier ski-club voit le jour à Glaris, sous l'impulsion de membres du Club alpin suisse (CAS), dont Christoph Iselin. D'autres ski-clubs s'organisent à partir du début du $\mathrm{XX}^{\mathrm{e}}$ siècle, notamment à Berne (1900), Zurich (1901), Davos (1903), Grindelwald (1902), Adelboden (1904) ou encore Wengen (1904) (Busset, 2016, p. 26). Si la liste n'est certainement pas exhaustive - un ski-club voit également le jour à la Vallée de Joux en 1905 (Cala, 2018) -, il est déjà intéressant de relever que, parmi les premiers clubs helvétiques, on retrouve trois ski-clubs de l'Oberland bernois et un ski-club des Grisons. À cela s'ajoutent encore des skiclubs britanniques, issus des mêmes régions, à l'image du Davos English Ski Club (1903). À cette période, l'Association suisse des clubs de ski est officiellement créée le 20 novembre 1904, et regroupe alors déjà 620 membres. Ce nombre croît rapidement pour atteindre 5192 en 1914 et plus du double, soit 11600 en 1929 (AFSS, Ski, 1929, pp. 12-13). Cela reste cependant limité en comparaison de disciplines telles que la gymnastique ou le football.

Durant cette période, le ski se démocratise, notamment sous l'impulsion de l'armée qui développe des cours de ski pour ses conscrits, comme le relève Anne Philipona Romanens (Philipona Romanens, 1999). De ce fait, il n'est pas surprenant de retrouver nombre de gradés au sein des structures du ski au sortir de la Première Guerre mondiale. Ainsi, la quasi-totalité des personnalités masculines citées dans le travail qui suit est gradée, et cet état de fait devra être examiné à l'avenir à l'instar de ce qui a été fait autour des élites économiques (Mach, David, Ginalski \& Bühlmann, 2016, pp. 55-58).

\subsection{La Lütschine, à la conquête de l'or blanc en terre bernoise}

Avant de nous intéresser aux institutions et aux logiques du développement touristique en Suisse, il faut relever que deux régions apparaissent comme essentielles et prédominantes dans la structuration des réseaux autour du ski helvétique, à savoir les Grisons et l'Oberland bernois, et notamment les vallées situées au-dessus d'Interlaken. En effet, jusqu'aux années 1950, ces deux régions sont centrales pour le développement touristique, comme le révèlent les statistiques hôtelières compulsées ${ }^{2}$.

La Lütschine est une région de l'Oberland bernois qui regroupe notamment les territoires de Mürren, Grindelwald, Wengen ou encore Lauterbrunnen. C'est dans ce petit coin de pays, au pied de la Jungfrau, qu'une partie de ce qui fait aujourd'hui encore la force de la discipline du ski en Suisse s'est construite. Les amateurs de ski connaissent cette région,

2 Les statistiques hôtelières suisses sont disponibles en ligne, il est ainsi possible d'obtenir rapidement des données sur les fréquentations cumulées dans les principales régions de Suisse. Cependant, des travaux approfondis sont encore à mener pour croiser l'ensemble des données. 
car elle accueille chaque année une étape de la Coupe du monde de ski alpin, à Wengen, où les meilleurs skieurs du monde s'affrontent sur les pentes du Lauberhorn. Cette course, aujourd'hui entrée dans l'histoire de la discipline, à l'image de la Streif de Kitzbühel, a été lancée par deux personnalités de la région en 1930, à savoir Christian Rubi, instituteur qui deviendra par la suite conseiller national socialiste, et Ernst Gertsch, propriétaire d'un magasin de sport et directeur des courses durant 40 ans (Gertsch \& Erb, 1969). Ces deux acteurs présentent ainsi déjà plusieurs caractéristiques intéressantes pour les réseaux du ski, entre l'organisation de compétitions, l'ancrage scolaire et la vente de biens sportifs.

Durant cette même période, en 1931, sont organisés à Mürren les premiers Championnats du monde de ski alpin. Cet événement a été rendu possible grâce notamment au travail de deux hommes : le Britannique Arnold Lunn, qui organise des voyages à Mürren pour ses concitoyens, et l'avocat Karl Dannegger, originaire de Thoune, aux portes de l'Oberland bernois. Ces deux hommes sont membres du Comité de direction de la FIS et ont longuement agi en faveur de la reconnaissance de la modalité alpine du ski par l'institution internationale, chose qu'ils réussissent à obtenir en 1930 (AFIS, Procès-verbal, Congrès, 1930, pp. 14-22). Après avoir imposé la modalité alpine et remis le chronomètre au centre des compétitions de slalom, Lunn continue à innover, notamment dans la technique du slalom (AMS, Annuaire de l'ASCS pour 1930, pp. $102-$ 111) et met en application ses idées lors de compétitions organisées à Mürren. Il est certain que la présence d'acteurs tels que Lunn et Dannegger dans l'Oberland bernois influence la structuration des réseaux du ski helvétique dans cette région. En effet, par son influence à la FIS et sa volonté de développer la technique et les compétitions de ski, Lunn a concentré l'attention des acteurs internationaux et nationaux sur la région du Lütschine. Par ailleurs, au sortir de la Première Guerre mondiale, Arnold
Lunn devient le Président du Federal Council of British Ski Club (ASCGB, The British Ski Yearbook, 1922, p. 158), et installe un peu plus les instances du ski britannique dans l'Oberland bernois. Ce qui n'est par ailleurs pas défavorable à ses activités professionnelles.

Afin de se rendre compte de l'importance que prend la Suisse sur la scène internationale du ski alpin, soulignons qu'entre 1931 et 1939, les championnats du monde sont organisés à quatre reprises sur le territoire helvétique et cela dans trois stations différentes, Mürren (1931 et 1935), Saint-Moritz (1934), et Engelberg (1938). Par ailleurs, la première édition des championnats du monde après la Seconde Guerre mondiale est encore organisée à Saint-Moritz en 1948. La décision est prise au Congrès de la FIS à Pau en 1946, avant même que la station grisonne ne soit désignée par le Comité international olympique pour accueillir les Jeux olympiques en 1948 (AFIS, Procès-verbal, Congrès, 1946, p. 28).

En plus d'avoir été un précurseur dans le domaine du ski, Arnold Lunn est le fondateur de plusieurs ski-clubs et notamment, en 1924, du Kandahar Ski-Club, basé à Mürren (Holt, 1992). Au sein de ce ski-club, on retrouve Elsa Roth qui n'est autre que la tante de Marc Hodler, le futur président de la FIS. Elsa Roth, originaire de Mürren, devient, dès 1934, membre du comité de l'ASCS où elle prend la responsabilité des finances et de la communication (AASCS, Rapport annuel, 1934, p. 193). Par la suite, elle est nommée secrétaire centrale à temps plein de l'association en 1939 et fera ses premiers pas à la FIS lors d'une réunion du Comité de direction à Lausanne, en pleine Seconde Guerre mondiale (AFIS, Rapport sur l'activité pendant la guerre, 1946), soulignant au passage le rôle décisif de la Suisse (et des dirigeants suisses) dans la poursuite des relations internationales sportives pendant les années de guerre (Vonnard \& Quin, 2019).

Nombre d'autres personnages importants du ski helvétique sont issus de cette petite région des alpes bernoises, comme par 
exemple Frit von Almen. Àl'instar de Christian Rubi, von Almen s'engage en politique où il connait un certain succès, avec notamment une élection au Conseil national, où il siège de 1935 à 1947. Issu d'une famille d'hôteliers de Lauterbrunnen, il s'investit beaucoup pour le développement touristique, et particulièrement celui du ski dans l'Oberland bernois (Quin \& Cala, 2019).

\subsection{Interassociation suisse pour le ski et Association des écoles suisses de ski, entre intérêts touristiques et sportifs}

Fritz von Almen compte, en 1934, parmi les fondateurs de l'AESS (AAESS, Procès-verbal, Commission régionale, le 4 mai 1934) où il représente justement les intérêts des milieux touristiques de l'Oberland bernois. Dans cette association sont représentés les Chemins de fer fédéraux (CFF), la Fédération suisse du tourisme (FST), les Postes, Téléphones et Télégraphes (PTT), la Société suisse des hôteliers - dont le président, Hermann Seiler, ancien conseiller d'État et conseiller national valaisan, très impliqué dans le développement du ski à Zermatt, participe de manière irrégulière aux séances de l'AESS (AAESS, Procès-verbaux du Comité, 1937) -, ou encore l'Office national suisse du tourisme (ONST). La présence de ces différentes sociétés touristiques peut paraître surprenante étant donné qu'il s'agit d'une association dont l'ambition est de fédérer les écoles de ski. Cependant, leur gestion, la qualité de formation des moniteurs ainsi que la méthode d'enseignement proposée aux touristes sont autant d'éléments qui peuvent avoir une influence sur la venue ou non de potentiels vacanciers et par conséquent sur le chiffre d'affaires des hôteliers et des entreprises de transport. De plus, l'AESS a également pour objectif d'unifier la communication des acteurs du ski et de créer un groupe d'intérêt afin de peser sur les pouvoirs publics (Offerlé, 1998). De ce fait, il n'est pas surprenant de retrouver différents acteurs touristiques au sein de l'AESS, notamment car, dès 1931, les CFF, les PTT et l'ONST unifient leurs efforts de propagande touristique, sous l'impulsion des autorités politiques fédérales (Garvaglia, 2011).

Au sein de l'AESS, on retrouve notamment les acteurs suivants : Christian Rubi, cité précédemment, Fritz Erb, né dans la Lütschine, qui est à la fois rédacteur en chef du grand quotidien spécialisé Sport et entraîneur des équipes suisses de ski aux Jeux olympiques de 1924 et 1928, ou encore Hans Bon qui prend la présidence du comité central (AAESS, Procèsverbal, Comité central, 13 septembre 1934). Ce dernier n'est pas bernois. Originaire des Grisons, il est propriétaire d'un hôtel luxueux à Saint-Moritz, le Suvretta House, et il préside également l'office du tourisme de la ville dès les années 1920 (Bon, 2010 ; Z’Graggen \& Comte, 2013). À côté de la Lütschine, l'Engadine est une région qui apparaît également prédominante, dans la construction des réseaux du ski helvétique. Ainsi, si le Ski-Club Alpina Saint-Moritz et le Ski-Club Davos sont respectivement le deuxième et le troisième skiclub de Suisse en termes de membres en 1930 (AASCS, Rapport annuel, 1930, pp. 198-199), ce sont surtout les activités des hôteliers qui ont développé la pratique des sports d'hiver et notamment du ski en Engadine (Seger, 2006). Il n'est dès lors pas étonnant de retrouver des hôteliers des Grisons actifs autour de la promotion du ski. La famille Bon en est un parfait exemple. Elle a notamment mis en service le premier téléski de la station de Saint-Moritz en 1935, dont le départ est situé à proximité directe de son hôtel : le Suvretta House (von Planta, 1978 ; von Planta, 1986). L'élection d'Hans Bon à la présidence de l'AESS est assez significative de l'importance de l'hôtellerie et des milieux touristiques en général au sein de l'AESS. Hans Bon, au-delà de son activité d'hôtelier, est aussi très actif dans la promotion de sa station, dont il préside à de nombreuses reprises l'Office du tourisme dans les années 1930, 1940 et 1950 (ACSM, Kur- und Verkehrsverein, procès-verbaux, 1930-1960), 
où il va construire son réseau. Dans le même temps, il est aussi membre du comité de la Société suisse des hôteliers, autre groupe d'intérêt, dont la trajectoire va croiser l'histoire du ski en 1931. En effet, ce groupe d'intérêt est responsable la publication de critiques explicites envers les méthodes d'enseignement du ski en Suisse, jugées alors peu favorables pour l'attractivité du pays, notamment face à l'Autriche (Quin, 2017).

Le contexte touristique en Suisse étant alors compliqué à cette période - les revenus de la branche baissent de plus de $60 \%$ entre 1929 et 1934 (Garvaglia, 2011, p. 76) -, le secteur hôtelier s'inquiète de voir les acteurs du ski autrichien développer une méthode d'enseignement unifiée et plus accessible aux touristes. Dès lors, la SSH presse les acteurs du ski helvétique, et en premier lieu l'ASCS, afin qu'ils mettent en place une méthode d'enseignement plus adaptée et unifiée pour l'ensemble du pays (Quin, 2017). Ces critiques débouchent une année plus tard sur la création de l'IASS qui a pour principaux objectifs : l'élaboration d'une nouvelle méthode d'enseignement éditée en français, en allemand et en anglais et la mise en place d'une nouvelle formation unifiée et certifiée pour les moniteurs. Cette association est dès le début liée aux milieux touristiques. Ainsi, parmi les membres fondateurs de l'IASS, nous retrouvons la Société suisse des hôteliers, ainsi que des représentants des gouvernements cantonaux des Grisons, de Berne et d'Uri (Pieth, 1983, p. 53). Cependant, l'influence des milieux touristiques y est moins importante qu'au sein de l'AESS, et ce sont des logiques plus " pédagogiques » et "sportives " qui semblent prédominer dans les premières années de l'IASS. Ainsi, le premier président, Christian Jost, est un instituteur de Davos. Il est accompagné au sein du comité formé de trois personnes par Franz Schuler, grison lui aussi et représentant de l'ASCS. Ce dernier est par ailleurs membre du Comité de direction de la FIS de 1934 à 1946 (AFIS, Procès-verbal, Congrès, 1934, p. 53). Tourisme, pédagogie et pratiques de compétitions se retrouvent donc en première ligne et servent d'axes pour la structuration des réseaux, autour des deux principaux espaces régionaux. Parmi les autres personnes actives au sein de l'IASS au cours des années 1930, il est intéressant de relever la présence de Hans Bon, de Fritz Erb, ou encore d'Othmar Gurtner qui est membre du comité de l'AESS (AIASS, Procès-verbal, Assemblée générale, 1935).

D'autres noms sont également à signaler, notamment celui de Hans Kasper, père de Peter Kasper, directeur de l'Office du tourisme de Saint-Moritz dès 1947 (Kasper, 1986), mais aussi grand-père de l'actuel président de la FIS, Gian Franco Kasper. Hans Kasper participe notamment au développement de la nouvelle méthode d'enseignement du ski au sein de l'IASS (Pieth, 1983, p. 53). Il était également responsable des cours pour moniteurs de l'IASS organisés à Saint-Moritz au début des années 1930 (AIASS, Procès-verbal, Comité central, 13 janvier 1933). Au-delà des liens familiaux et personnels pouvant exister, force est de constater que les réseaux du ski sont aussi des réseaux basés sur des noyaux familiaux très puissants et des « dynasties » de dirigeants. Peter Kasper est d'ailleurs très proche de la famille Bon - et notamment Hans Bon avec qui il partage des séjours en Angleterre dans les années d'après-guerre, rencontrant alors notamment Winston Churchill (Kasper, 1986, p. 13).

Les acteurs du ski helvétique, qu'ils soient membre de l'ASCS, de la FIS, de l'IASS ou de l'AESS, sont en relations régulières entre eux à travers les différentes structures où certains assument des fonctions multiples. Ainsi, si l'IASS semble être moins influencée que l'AESS par les milieux touristiques, des liens importants existent tout de même avec l'hôtellerie.

Au sortir de la Seconde Guerre mondiale, le ski suisse apparaît dès lors comme incontournable, cela d'autant plus que les acteurs du ski helvétique innovent. Les premiers slaloms 
géants sont en effet organisés en Suisse, plus précisément à Zermatt, en mars 1946. Ils sont alors prévus pour les femmes, en remplacement de la descente, jugée trop dangereuse (AFIS, Procès-verbal, Congrès, 1946). Par ailleurs, durant la guerre, la Suisse a continué, à l'inverse de ses voisins directs, son développement infrastructurel, notamment au niveau des transports et des remontées mécaniques, ce qui renforce son attractivité (Humair \& Tissot, 2011). Dans le même temps, la Suisse va définitivement imposer son statut par l'intermédiaire de trois événements majeurs : la nomination de Saint-Moritz comme ville-hôte de l'édition de 1948 des Championnats du monde de ski ; la désignation de la même ville par le CIO pour accueillir les Jeux olympiques d'hiver de 1948 et l'élection d'un citoyen suisse à la présidence de la FIS. De fait, ces divers éléments ont permis un développement certain de la discipline au niveau national, ce qui a également contribué à la valorisation de l'image du ski helvétique sur le plan international, à la fois en termes de résultats sportifs mais aussi pour la visibilité de la place touristique.

Les changements structurels mis en place durant l'entre-deux-guerres vont également apporter des résultats en ce qui concerne le développement touristique des stations, même si ce développement n'est pas visible partout et devra être soutenu par des mesures plus directes des pouvoirs publics, notamment en subventionnant le coût des heures de cours dans les écoles de ski pour les touristes (Quin \& Cala, 2019).

\section{UN OR BLANC... SONNANT ET TRÉBUCHANT (1951-1964)}

À l'occasion du congrès de la FIS de 1951, organisé à Venise, le délégué suisse Marc Hodler est choisi pour la présidence de l'organisation (AFIS, Procès-verbal, Congrès, 1951, p. 24). Il devient alors le troisième président de la fédération après le lieutenant-général suédois Ivar Holmquist et le colonel norvégien Nicolai Ramm Ostgaard. Dans la mesure où la «présidence » de la FIS est constituée d'un président et d'un secrétaire général (FIS, Statuts, 1946, art. 16) et que les usages sont ainsi depuis l'entre-deux-guerres, le président Hodler va alors être accompagné dans sa fonction par un secrétaire du même pays que lui, en la personne de Arnold Kaech. Le siège de la FIS va alors déménager dans les beaux quartiers de la rive droite de l'Aare à Berne, dans les locaux de l'étude d'avocats de la famille Hodler. Arnold Kaech est alors directeur de la toute jeune École fédérale de sport de Macolin depuis 1946, mais il est surtout un ancien skieur émérite et un fonctionnaire actif dans l'administration militaire, une tutelle dont l'importance pour le développement du ski est déjà bien avérée (Peiffer, 2005), tout particulièrement en Suisse (Giuliani, 2001 ; Busset, 2016).

De fait, l'élection de Marc Hodler à la FIS est soutenue par une majorité de 20 voix (contre 16 voix pour une candidature française) et repose sur des liens déjà anciens de la famille au sein des cercles dirigeants du ski, et notamment autour de la figure d'Arnold Lunn (Holt, 1992). En effet, Elsa Roth est une amie proche de la famille Lunn et entretient notamment une correspondance avec la compagne de Lunn elle-même. Les deux familles sont en outre impliquées dans les premières courses de ski alpin organisées dans la région de la Lütschine, autour du Kandahar Ski-Club notamment (Lunn, 1969).

Dans les années 1950 et sous l'impulsion de Marc Hodler, le ski alpin va rapidement obtenir une légitimité plus forte dans le cadre de la FIS, confirmant ainsi l'influence de la Suisse sur la scène internationale du ski. En effet, dans les années 1950, les activités d'Elsa Roth sont nombreuses et décisives, depuis la création du système de comptage des points lors des saisons internationales de ski, jusqu'à sa proposition - assez avant-gardiste - d'élaborer un système de balisage unifié des pistes 
au niveau international (AFIS, Procès-verbal, Congrès 1946, pp. 43-44).

Dans les réseaux suisses, les années 1950 vont correspondre au développement d'une approche plus "économique " du ski alpin, dont l'activité devient assurément un argument touristique majeur dans de nombreuses stations de sports d'hiver, de l'Oberland bernois aux Grisons, en passant par Zermatt (Guex, 2016). Ces dynamiques peuvent s'observer à travers l'arrivée dans les cercles dirigeants de nouveaux promoteurs du tourisme et de commerçants liés à l'industrie des biens sportifs (ici notamment le matériel technique et les vêtements), les fabricants de matériel de sport intégrant en effet officiellement l'AESS en 1945 (AAESS, Procès-verbal, Comité central, 22 juin 1945, p. 1). S'il est trop réducteur de considérer ici les vendeurs d'articles de sport comme des vecteurs uniques de nouveaux intérêts économiques - la plupart sont en outre moniteurs de ski et d'anciens skieurs d'élite -, il n'est plus possible de ne pas prendre ces nouveaux intérêts en compte à ce stade.

Dans le comité de l'IASS de 1951, sous la présidence de Hugo Brandenberger - dont l'activité a démarré dès les années 1930 -, nous retrouvons notamment Marc Hodler qui cumule les fonctions dans les réseaux du ski, jusqu'à constituer lui-même le cœur de ce réseau - ou Hans Leutert mais aussi Jack Ettinger. Ce dernier est une figure importante du ski dans les Grisons. Ancien skieur de l'équipe nationale dans l'entre-deux-guerres, directeur du magasin de sport familial à Davos, Jack Ettinger représente une nouvelle génération de dirigeants, dont l'intérêt pour le développement du ski en Suisse est couplé à un intérêt commercial plus objectif et notamment celui de faire croître les activités de sa fabrique de skis. Si celle-ci ne va pas se développer au-delà des frontières des Grisons, elle engage Adolf Attenhofer dès 1917, lequel développera sa propre marque à partir de 1924, avec un succès international (Hitz, non daté ; Triet, 2001). En 1952, le comité de l'IASS voit aussi arriver
Adolf Odermatt, directeur de l'Hôtel-Bellevue Terminus à Engelberg et donc très directement lié à des intérêts touristiques et économiques, après avoir notamment dirigé l'école de ski de la station dès 1950 (Hôtel-Revue, 21 décembre 1950, p. 15), ainsi que Christian Rubi, dont l'implication est déjà forte depuis les années 1930 .

$\mathrm{Au}$ sein de l'AESS, le réseau des dirigeants va aussi évoluer. En 1951, le président de l'AESS est le docteur Decurtins, directeur de l'hôtel Schweizerhof à Lenzerheide. Il s'est notamment engagé avec Christian Rubi, afin de mettre en place le subventionnement fédéral, pour les touristes, des heures de cours dans les écoles de ski (Quin \& Cala, 2019). En cette année 1951, le système de subventionnement semble fonctionner pour « combattre la réputation de vie chère de la Suisse [et] pour obtenir un tourisme plus libre " (AAESS, Procès-verbal, Assemblée générale, 1951, p. 3). Dans son rapport annuel fait à l'occasion de l'assemblée générale de 1952, Decurtins réitère du reste ses demandes : « En Autriche et ailleurs, l'enseignement du ski est encouragé par des moyens bien plus considérables que chez nous. C'est pourquoi le président adresse aux autorités un pressant appel pour le maintien de leur appui financier » (AAESS, Procèsverbal, Assemblée générale, 1952, p. 1).

De fait, les discussions ne portent cependant pas uniquement sur des aspects purement économiques et à l'instar des débats du début des années 1930 (Quin, 2017), au milieu des années 1950, une querelle intense éclate entre les milieux dirigeants du ski - IASS et AESS en tête - et l'instructeur Josef Dahinden, lequel adresse de nombreux reproches à la méthode officielle véhiculée depuis les années 1930, et notamment sur le virage qui y est enseigné (Pieth, 1983, pp. 33-34). S'il faudra prochainement approfondir les dynamiques de ces débats, les discussions révèlent notamment plusieurs contradictions encore très fortes dans le système sportif suisse. Ainsi, dans son rapport annuel pour l'hiver 1954-1955, Hugo 
Brandenberger souligne qu'il est possible d'écarter

"le reproche affirmant que la technique enseignée en Suisse est la cause de l'insuccès de nos coureurs. La technique n'est que l'un des facteurs de la compétition. L'esprit combatif, la condition physique, la possibilité de réagir aussi bien physiquement que mentalement, et la chance enfin, sont également des facteurs d'aussi grande importance.

Les performances, aujourd'hui, ne dépendent pas seulement des qualités physiques, mais de la possibilité de se vouer complètement et sans condition à son sport. Le sportif devra suivre une ligne de conduite dont bien souvent sa famille et son travail pâtiront [...] (AIASS, Rapport annuel, 1954-1955, p. 6).

Toujours en 1954, Arnold Kaech rejoint le comité de l'IASS, où il retrouve donc Marc Hodler, dont il est aussi le secrétaire général à la FIS. Du reste, en ce milieu des années 1950, le système sportif suisse va connaitre un premier électrochoc dans le cadre des discussions entourant l'envoi d'une délégation à Melbourne suite à l'invasion de Budapest. Ce que certains ont qualifié de «boycott » est en fait une "non-participation ", liée au fonctionnement encore assez lent du système helvétique (Tonnerre \& Quin, 2017). Pour les sports d'hiver, les tensions géopolitiques sont encore moindres, au-delà des débats qui ont entouré la réintégration de l'Autriche, de l'Allemagne et du Japon dans les organisations internationales (Dichter, 2017). Néanmoins, depuis son élection à la présidence de la FIS, le président Hodler rappelle inlassablement les termes de l'accord trouvé entre son institution et le CIO juste après la guerre, permettant notamment aux moniteurs de ski d'être qualifiés au-delà des règlements olympiques sur l'amateurisme (AFIS, Procès-verbal, Congrès, 1946, pp. 14-15). Inspirés par l'exclusion des "moniteurs » suisses lors des Jeux olympiques de 1936, cette démarche de Marc Hodler témoigne d'une forme de modernité dans les milieux du ski vis-à-vis des nouveaux enjeux économiques du sport international.

En 1955, Peter Kasper devient le président de l'AESS, il est alors le troisième "Grisons " à officier à ce poste, même s'il n'est pas directeur d'hôtel comme ses prédécesseurs (AAESS, 1984, p. 17). Il est en revanche directeur de l'Office du tourisme de Saint-Moritz et a, selon de nombreuses sources concordantes, joué un rôle important dans l'organisation des Jeux olympiques de 1948 (Kasper, 1986, p. 18) et dans le développement de la station grisonne depuis les années 1930.

À l'orée des années 1960, une fratrie de l'Oberland bernois va émerger sur l'avantscène du ski suisse : les Glatthard. En 1961, Arnold Glatthard, alpiniste chevronné, intègre le comité de l'IASS lorsque l'institution élargit sa composition pour obtenir " une représentation plus équitable des différentes régions du pays » (AIASS, Rapport annuel, 1960-1961, p. 4). Arnold Glatthard est notamment le fondateur de l'Institut d'alpinisme de Rosenlaui, première école d'alpinisme au monde (Bernet, 2007). En parallèle, il est aussi commerçant à Meiringen, où il est très intégré dans la vie politique locale. Ancien entraineur de l'équipe suisse de ski, et lui-même ancien vainqueur au Lauberhorn en 1935, il semble posséder un profil à la croisée des enjeux du ski helvétique. Dès la fin des années 1950, c'est pourtant le petit frère, Karl, qui va occuper les premiers rôles. De trois ans le cadet de Arnold, il constitue un exemple hors norme des relations que vont tisser les élites du sport suisse dans les sphères politiques, économiques et militaires à un moment clé de l'histoire du sport helvétique (Quin, 2018). S'il est colonel au sein de l'armée suisse - élément des plus important si l'on souhaite réaliser une carrière dans l'administration ou dans le sport helvétique -, c'est aussi par son mariage que Karl Glatthard va atteindre des fonctions de responsabilité dans le cadre de l'entreprise de construction « Wahlen » basée à Berne, dont il épouse l'héritière - Margarita Elisabeth Wahlen (Stettler, 
2007). Membre du conseil de la ville de Berne entre 1956 et 1967, il est aussi élu au Grand Conseil bernois de 1962 à 1967, avant de devenir conseiller national entre 1967 et 1971. Surtout, il s'implique très rapidement dans différents comités et institutions sportives, notamment à l'Association nationale d'éducation physique (ANEP). Président de la Fédération suisse de ski dès 1963 , il va y introduire une gestion plus moderne, inspirée par les modèles de gouvernance qu'il connait dans son milieu professionnel, avec notamment une distinction plus claire entre les tâches administratives du secrétariat et les domaines plus techniques liés au suivi des athlètes, ce que nous observerons autour du duo Elsa Roth-Adolf Ogi dès 1964. Glatthard est aussi membre de la commission de sport militaire, de la CFGS et de la commission militaire du ski. Karl Glatthard et Marc Hodler forment alors l'axe fort des réussites du ski suisse dès la seconde moitié des années 1960. Dans le même temps, la réussite économique du tourisme hivernal suisse à la fin des années 1950 et au début des années 1960 est croissante. Selon la Nouvelle Revue de Lausanne, le ski en Suisse connaît un engouement florissant :

" Le ski connaît un engouement, une faveur populaire qui vont chaque année [en] grandissant. Et ce n'est certes pas cette saison, où chacun attend avec impatience les exploits de l'élite des skieurs mondiaux, réunis aux Jeux olympiques d'Innsbruck dès le 29 janvier prochain, qu'un coup de frein sera donné au mouvement. D'autant plus que l'ambiance est toujours à la haute conjoncture " (Nouvelle Revue de Lausanne, 29 novembre 1963).

Malgré cela, les Jeux olympiques d'Innsbruck vont être le théâtre d'un échec qui ne sera pas sans conséquence pour le ski et de manière générale pour le sport suisse.

\section{De L'ÉCHeC d'InNSBRUCK (1964) À LA descente de Berchtesgaden (1967)}

Dans l'histoire des sports en Suisse, et particulièrement du ski, l'année 1964 doit être considérée comme un tournant, en raison notamment de l'échec de la délégation lors des Jeux olympiques d'hiver à Innsbruck les athlètes helvétiques n'obtiennent aucune médaille - et de toutes les transformations que vont entamer les autorités sportives, politiques, économiques et pédagogiques à la suite de cette " débâcle " (Quin, 2018 ; Hug, 2018). En effet, ces Jeux olympiques sont présentés par la presse comme un affront au sein de la population, et vécus comme tels par certains élus et dans de nombreux cercles dirigeants du sport suisse. Comme l'indique le conseiller fédéral Paul Chaudet dans son éditorial publié le 9 février 1964 à la Une du Sport, le sport suisse d'élite doit désormais bénéficier d'un meilleur soutien de la part de l'État (Sport, 9 février 1964) et dès le 17 février 1964, plusieurs questions sont déposées au Conseil national, comme celle de Weisskopf, qui cible notamment le biathlon.

"Les résultats de tir à l'arme de guerre dans la discipline de ski de fond ont été lamentables pour des fils de Tell [...]. On peut craindre que des conclusions erronées ne soient tirées au sujet de notre valeur militaire. Le Conseil fédéral est-il disposé à prendre à l'avenir des mesures pour que le choix et l'entraînement des délégations suisses aux championnats militaires internationaux soient faits d'une façon soigneuse, méthodique et complète [...] » (CNSE, Question Weisskopf, 17 février 1964).

Si la prise de position du conseiller national Weisskopf souligne plusieurs enjeux de l'événement, elle cherche surtout à placer les débats autour de la question militaire, du soutien au sport d'élite et donc des prérogatives du conseiller fédéral Paul Chaudet. Cet élément du subside est d'ailleurs aussi au cœur d'une autre question posée par le conseiller 
national Germanier, qui se demande s'il ne "serait pas indiqué, notamment, de confier au département militaire, dans le cadre de son budget, la préparation à certaines disciplines sportives d'intérêt national » (CNSE, Question Germanier, 17 février 1964). De fait, les débats cherchent donc à discuter l'organisation générale du système sportif suisse, et notamment des soutiens " politiques " pour le sport d'élite et pour le sport de masse, alors que n'existe encore aucune base légale pour l'encouragement du sport.

L'une des solutions évoquées alors est la reprise par la Confédération des cours dédiés au "sport de masse ", de manière à libérer l'ANEP de ces obligations. De même, l'École de Macolin pourrait être plus directement mise à disposition des sportifs de pointe, soit avec ses moniteurs, soit à travers des facilités d'hébergement et d'entraînement (CNSE, Compte rendu de la séance entre Paul Chaudet et l'ANEP, 16 avril 1934, p. 1). De fait, l'ensemble des fédérations membres de l'ANEP sont alors interrogées pour connaître leurs besoins et leurs souhaits en termes d'amélioration du fonctionnement du système sportif. Chacune peut exposer ses besoins et les différents types de soutien dont elles pourraient bénéficier pour mieux encadrer ses meilleurs athlètes. Ainsi, dans son courrier daté du 22 mai 1964, Karl Glatthard, le président de la Fédération suisse de ski, souligne qu'il est compliqué pour ses meilleurs skieuses et skieurs de s'entraîner dans les meilleures conditions quand celles-ci et ceux-ci doivent encore cumuler leur sport et une activité professionnelle (CNSE, Lettre de Karl Glatthard à l'ANEP, 22 mai 1964). De fait, le ski et le football sont sans doute les sports où un semi-professionnalisme peut exister, mais il reste encore très limité et globalement les mentalités helvétiques sont encore réticentes à l'idée de permettre l'émergence d'un vrai professionnalisme dans le sport (Vonnard, Berthoud \& Quin, 2018). Cette position est résumée dans un courrier du directeur de l'Administration militaire fédérale, Arnold
Kaech, qui indique qu'il ne peut «s'agir pour nous de suivre certains exemples de l'étranger et d'étatiser le sport ou de créer des "amateurs d'État” " (CNSE, Lettre d'Arnold Kaech à l'ANEP, 17 juin 1964), des propos repris à la Une du journal Sport quelques jours plus tard sous la formule : " La Suisse a-t-elle besoin de gladiateurs? " (Sport, 19 juin 1964). À la suite de ces démarches, une " commission d'étude pour le soutien aux sportifs de pointe " est mise en place le 4 juillet 1964, avec une première séance planifiée le 14 juillet 1964. Parmi les membres de cette commission, on retrouve plusieurs dirigeants actifs depuis plusieurs années ou décennies dans les institutions du sport suisse, et dont la sélection repose avant tout sur les compétences dans le sport de pointe. Le comité de pilotage est ainsi composé de :

- «Walter Siegenthaler (président de l'ANEP)

- Raymond Gafner (président du COS)

- Louis Perrochon (membre de la Commission fédérale de Gymnastique et de Sport)

- Ernst Hirt (directeur de l'École fédérale de Sport)

- Ernst Thommen (directeur de la Société du Sport-Toto)

- Marc Hodler (membre du CIO et président de la FIS)

- Dr. Gottfried Schönholzer (président de la commission médico-sportive de l'ANEP)

- Rolf Bögli (secrétaire central de l'ANEP) » (CNSE, Liste des membres de la commission d'étude de l'ANEP sur le sport de pointe).

Les membres du comité de pilotage ont alors la charge de se prononcer sur les membres potentiels - tels que Ulrich Frey, Karl Glatthard ou Kaspar Wolf, ainsi que des journalistes des principaux journaux helvétiques -, susceptibles d'intégrer les sous-commissions imaginées pour réformer le soutien aux athlètes de pointe. Après quelques discussions supplémentaires, l'ANEP décide durant son Assemblée des délégués du 14 novembre 
1965 de créer un « Comité national pour le sport d'élite ", lequel est considéré davantage comme une "communauté de travail " plus que comme une nouvelle institution (ANEP, PV-Assemblée délégués pour 1965). Le modèle ici est davantage celui de l'inter-association, à l'image de ce qui existe pour le ski depuis les années 1930 (Quin, 2017), supposée fédérer les volontés de ses membres. De fait, les différentes organisations et institutions du sport suisse doivent y être représentées : l'ANEP, le COS, la CFGS, l'EFGS, les fédérations sportives, mais aussi des organisations comme la Société du Sport-Toto, l'institut de recherche de l'EFGS et la représentation suisse au sein du CIO (Quin, 2018). C'est au titre de ces dernières fonctions, il est membre du CIO depuis 1963, que Marc Hodler participe d'emblée à ces dynamiques, représentant aussi les intérêts du ski dans ces discussions.

Si le nouveau comité doit favoriser le travail inter-fédérations, les dynamiques semblent alors aussi démarrer au sein des fédérations elles-mêmes, et notamment au sein de la Fédération suisse de ski, l'une des principales cibles des critiques, après l'échec de 1964. Au sein de la FSS, l'administration jusqu'ici dirigée par Elsa Roth va diviser ses tâches entre l'administration pure - qui reste sous la direction d'Elsa Roth - et la technique qui va revenir à Adolf Ogi dès 1964. Ce dernier intègre alors la Fédération sur le souhait d'Elsa Roth, mais avec assurément l'accord de Karl Glatthard, président de la FSS depuis 1962 et originaire de Meirigen, dont Adolf Ogi était alors directeur de l'Office du tourisme. D'emblée, Adolf Ogi occupe alors des fonctions de coordinateur des équipes nationales, avec la mission de repositionner le ski suisse au plus haut niveau des hiérarchies alpines (et mondiales). Dans le même temps, Karl Gamma devient directeur de l'AESS en 1964, en succession de Christian Rubi. Gamma, à l'instar de Glatthard, va devenir un grand nom du ski suisse, et plus largement du sport suisse, avec des implications multiples : "instructeur en chef de la FSS (1963-1992), directeur de l'AESS (1964-1992), président de la commission technique de l'IASS (1965-1992), président de l'Association internationale des moniteurs de ski (1971-1987) » (Lerch, 2005), continuant ainsi une tradition helvétique d'engagement à plusieurs niveaux.

\section{ÉPILOGUE}

Dans les faits, les réseaux qui se sont mis en place autour du ski en Suisse à partir des années 1930 ont favorisé le développement de la discipline sous de nombreux aspects. L'analyse qui en a été faite n'est assurément pas exhaustive, mais permet déjà de poser quelques jalons autour des dynamiques qui ont favorisé le développement du ski, à la fois sur le plan pédagogique, sportif et bien entendu économique. De fait, si la pratique du ski a connu un essor important, durant la période étudiée, celui-ci est en grande partie dû au fait que les intérêts de personnalités issues des milieux touristiques, sportifs, politiques et pédagogiques se sont rencontrés au sein d'un réseau important et polarisés autour de deux régions majeures pour le ski helvétique et international, à savoir l'Oberland bernois et les Grisons. L'influence de ce réseau à travers la FSS, l'IASS, l'AESS et la FIS a permis à la discipline de construire les bases de son développement sportif et touristique. Si l'analyse s'est concentrée sur les deux régions précitées, il ne faut pas occulter le travail réalisé dans d'autres contrées helvétiques, et notamment le Valais (Zermatt, Crans-Montana, Laax ou Saastal), la Suisse centrale (Engelberg) ou le Canton de Vaud (Les Diablerets, Villars ou la Vallée de Joux). Cependant, si des réseaux d'importance existent également dans ces régions, ils n'ont pas une influence aussi prépondérante que ceux issus de l'Oberland bernois et des Grisons à la période étudiée.

Durant les années 1960, ce réseau du ski helvétique, structuré autour de Marc Hodler, est toujours influent. Cette décennie est 
cependant marquée par d'importants changements pour le ski international et la montée en puissance de nouveaux acteurs issus notamment de la presse et des médias. La fin des années 1960 voit en effet la création d'un circuit international. Sous l'impulsion des dirigeants du journal L'Équipe, la FIS instaure la Coupe du monde de ski à partir de la saison 1967 (Montérémal, 2007, pp. 117118). La presse et les médias sont alors directement intégrés aux discussions et prennent même une place prépondérante au sein de la structure dirigeant l'organisation de la Coupe du monde. En 1967 à Beyrouth, le Congrès de la FIS valide en effet, sur proposition du Comité et notamment de son président Marc Hodler, la création d'un «FIS Alpin World Cup Board ", composé de membres de la FIS, mais également de deux journalistes, en l'occurrence un membre de l'Association internationale de la presse sportive et un autre membre de l'Association internationale des journalistes du ski (AFIS, Procès-verbal, Congrès, 1967, pp. 87-88). L'intégration de représentants des médias au sein même d'une structure de la FIS est certainement un tournant. Cela dénote une volonté d'augmenter et de développer la médiatisation du ski, à une période où la mondovision est en plein essor et où la télévision se démocratise (Alves, 2007, pp. 28-32). Si le calendrier international comporte depuis plusieurs années des dates régulières où les meilleur.e.s skieurs et skieuses du monde peuvent se mesurer, la Coupe du monde va changer en profondeur le paysage du ski international. L'industrie du tourisme hivernal dans les Alpes profite alors d'une nouvelle vitrine, qui vient s'ajouter à un contexte social, politique et économique favorable à son développement. Comme le relève Montérémal, l'environnement socio-économique des années 1960 « est profondément marqué par la progression du pouvoir d'achat des « classes moyennes » et leur accès à la consommation de masse. C'est le moment où les stations de ski de troisième génération, dites "stations intégrées ", sont construites en haute altitude. La pratique [du ski] commence à se démocratiser» (Montérémal, 2007, p. 117).

La Suisse se retrouve, sans surprise, au centre du nouveau calendrier international avec l'organisation de trois étapes au début du mois de janvier dans l'Oberland bernois, à Adelboden, Grindelwald et Wengen. C'est le pays qui accueille, avec les États-Unis, le plus de compétitions lors de la première édition de la Coupe du monde de la FIS. Les dates retenues sont par ailleurs excellentes pour les milieux touristiques helvétiques qui peuvent profiter de l'impact médiatique de la Coupe du monde dès le début de la saison hivernale. De nouvelles étapes sur sol suisse - SaintMoritz, Les Diablerets, Crans-Montana, Laax, Meiringen, Mürren, Ebnat-Kappel, Villars ou encore Arosa - apparaissent dans le calendrier à partir des années 1970. Dans ce cadre, il serait intéressant d'analyser quel rôle les réseaux du ski suisse ont joué dans l'acquisition d'étape de la Coupe du monde par ces stations, dans un contexte de multiplication des candidatures suisses, notamment des Grisons ou de l'Oberland bernois, pour les Jeux olympiques d'hiver (Lacotte, Kiuri \& Stricker, 2017, p. 71 et suivantes). Il est certain que cette prépondérance des étapes suisses sur le circuit mondial - en 1977, il y a sept étapes dans les Alpes suisses - a participé au développement de l'image «montagnarde » et « hivernale » du tourisme helvétique. Il est probable - cela doit tout de même être analysé - que cela ait aussi favorisé l'industrie du tourisme alpin en Suisse et donc une part prépondérante des acteurs du ski helvétique.

\section{BibLIOGRAPHIE}

\section{Sources}

Archives de la Fédération internationale de ski (AFIS) : Congrès, procès-verbaux (1924-1964).

Archives de l'Association suisse des clubs de ski (AASCS) : Rapports annuels (1923-1964). 
Archives de l'Interassociation suisse pour le ski (AIASS) : Assemblée des délégués, procès-verbaux (1933-1964). Rapports annuels (1933-1964).

Archives de l'Association des écoles de ski suisses (AAESS) : Assemblée des délégués, procès-verbaux (1934-1964). Rapports annuels (1934-1964).

Archives du Comité national pour le sport d'élite (ACNSE) : Documents relatifs à la création du CNSE (1964-1965).

Archives de la Commune de Saint-Moritz (ACSM) : Kurund Verkehrsverein, procès-verbaux (1929-1965).

\section{Littérature secondaire}

Alves, P. (2007). L'Union européenne de Radiodiffusion, 1950-1969. Une approche internationale et communautaire de la télévision. Bulletin de l'Institut Pierre Renouvin, 26(2), 19-34.

Arnaud, P. (1991). Olympisme et sports d'hiver : les retombées des Jeux olympiques d'hiver de Chamonix 1924. Revue de géographie alpine, 79(3), $15-36$.

Association des écoles suisses de ski (1984). 50 ans de l'AESS. Andermatt: AESS.

Bernet, P. (2007). Glatthard, Arnold. Dictionnaire historique de la Suisse. http://www.hls-dhs-dss.ch/ textes/f/F48207.php.

Bon, D. R. (2010). Geschichte einer Erbschaft. Regensberg : David R. Bon.

Busset, T. (2005). La relation travail-loisirs à travers l'avènement des sports d'hiver dans les Préalpes et Alpes de Suisse occidentale. Société suisse d'histoire économique et sociale, 20, 263-272.

Busset, T. (2016). La diffusion du ski en Suisse jusqu'à l'entre-deux-guerres. Traverse, 23(1), 25-36.

Cala, S. (2018). Ski et tourisme dans la Vallée de Joux, à la croisée des intérêts sportifs et touristiques (18991939). Entreprises et histoire, 93, 62-74.

Dichter, H. (2017). We have allowed our decisions to be determined by political considerations : the early Cold War in the International Ski Federation. Sport in History, 37(3), 290-308.

Engel, P. (2013). The Discursive Construction of National Identity through the Swiss Magazine Ski Before World War I. The International Journal of the History of Sport, 30(6), 598-616.

Garavaglia, R. (2011). Le rôle des pouvoirs publics dans la promotion touristique suisse à l'étranger : le cas de la France des années 1930. In C. Humair $\&$ L. Tissot (dir.). Le tourisme suisse et son rayonnement international (XIX'e-XXe siècles) (pp. 75-88). Lausanne : Antipodes.
Gertsch, E. \& Erb, K. (1969). 40 Jahre. Internationales Lauberhorn-Skirennen Wengen. Interlaken : Schlaefli.

Gigase, M., Humair, C. \& Tissot, L. (2014). Le tourisme comme facteur de transformations économiques, techniques et sociales $\left(19^{\mathrm{e}}-20^{\mathrm{e}}\right.$ siècles). Neuchâtel : Alphil.

Giuliani, M. (2001). " Starke Jugend - Freies Volk ». Bundesstaatliche Körpererziehung und Gesellschaftlische Funktion von Sport in der Schweiz (1918-1947). Berne : Peter Lang.

Guex, D. (2016). Tourisme, mobilités et développement régional dans les Alpes Suisses : mise en scène et valeur territoriale. Montreux, Finhaut et Zermatt du XIX siècle à nos jours. Neuchâtel : Alphil.

Guex, D., Roy, J. \& Sauthier G. (2012). La trajectoire historique du développement touristique de Montreux entre 1850 et 2010. Sion : Institut universitaire Kurt Bösch.

Hitz, L. (non daté). A Glance at Switzerland's skiing history, International Skiing History Association, online magazine, https://skiinghistory.org/history/ glance-switzerlands-skiing-history.

Hoibian, O. (2006). Sociogenesis of a Social Field: The Cultural World of Mountaineering in France from 1870 to 1930. International Review for the Sociology of Sport, 41(3-4), 339-355.

Holt, R. (1992). An Englishman in the Alps : Arnold Lunn, amateurism and the invention of alpine ski racing. The International Journal of the History of Sport, 9(3), 421-432.

Hug, B. (2018). Mesurer le corps performant à l'occasion des " jeux olympiques sans oxygène ". Perspectives helvétiques sur le rôle de la médecine du sport autour des jeux Olympiques de 1968. In G. Quin \& A. Bohuon (dir.). 1968, le sport fait sa révolution à Mexico (pp. 47-90). Paris : Glyphe.

Humair, C. \& Tissot, L. (2011). Le tourisme suisse et son rayonnement international "Switzerland, the playground od the world ». Lausanne : Antipodes.

Humair, C., Guex, S., Mach, A. \& Eichenberger, P. (2012). Les organisations patronales suisses entre coordination économique et influence politique. Vingtième siècle. Revue d'histoire, 115(3), 115-127.

Humair, C., Gigase, M., Lapointe Guigoz, J. \& Sulmoni, S. (2014). Système touristique et culture technique dans l'arc lémanique : analyse d'une success story et de ses effets sur l'économie régionale (1852-1914). Neuchâtel: Alphil.

Kasper, C. (1986). Peter Kasper. Grand Old Man des Tourismus. Samedan : Engadin Press.

Lacotte, U., Kiuri, M. \& Stricker, C. (2017). Olympische Spiele in der Schweiz. Thoune : Werd Verlag. 
Lemercier, C. (2005). Analyse de réseaux et histoire. Revue d'histoire moderne et contemporaine, 52(2), 88-112.

Lerch, H. (2005). Gamma, Karl. Dictionnaire historique de la Suisse. http://www.hls-dhs-dss.ch/textes/f/ F14056.php.

Lunn, Sir A. (1969). The Kandahar Story. London : George Allen \& Unwin.

Mach, A., David, T., Ginalski, S. \& Bühlmann, F. (2016). Les élites économiques suisses au XXe siècle. Neuchâtel : Alphil.

Montérémal, G. (2007). L'Équipe: médiateur et producteur de spectacle sportif (1946-1967). Le Temps des médias, 9(2), 107-120.

Offerlé, M. (1998). Sociologie des groupes d'intérêts. Paris : Montchrestien.

Peiffer, L. (2005). Vom Soldatensport zum Volksport. Das Militär als Katalysator der Populasierung des Skilaufs. In M. Herzog (dir.). Skilauf - Volksport - Medienzirkus. Skisport als Kulturphänomen (pp. 69-94). Stuttgart: Kohlhammer.

Pieth, F. (1983). 50 ans. Interassociation suisse pour le ski. Berne : IASS.

Quin, G. (2017). De la cure d'air à l'or blanc. Une "Interassociation suisse pour le ski " face aux enjeux de l'essor du ski en Suisse (années 1920-années 1960). Histoire des Alpes, 22, 135-155.

Quin, G. (2018). Aux frontières de la médecine, de la diplomatie et du sport en Suisse. La création du Comité National pour le Sport Elite (1956-1972). In G. Quin \& A. Bohuon (dir.). 1968, le sport fait sa révolution à Mexico (pp. 91-117). Paris : Glyphe.

Quin, G. \& Cala, S. (2019). Structuration et promotion du ski en Suisse (années 1920-années 1960). Est-ce l'engagement des dirigeants ou les pentes des massifs qui expliquent l'essor du ski alpin helvétique? In P. Vonnard, G. Quin \& C. Jaccoud (dir.), Des réseaux et des hommes. Participation et contribution de la Suisse à l'internationalisation du sport (1912-1972) (pp. 99-133). Neuchâtel : Alphil.

Schut, P.-O. (2013). Sport as a Major Player in the Development of Tourism : The History of Mountaineering in the Pelvoux Massif, France, from 1861 to 1914. The International Journal of the History of Sport, 30(12), 1329-1350.

Schut P.-O. \& Levet-Labry E. (2014). Les relations entre tourisme et sport autour des Jeux olympiques de 1924. Staps, 105, 37-49.

Seger, C. (2006). Wintersport im Grand Hotel : Strategien einer Inszenierung - das Oberengadin zwischen 1886-1914. In T. Busset \& M. Marcacci (dir.). Pour une histoire des sports d'hiver (pp. 35-49). Neuchâtel : CIES.

Stettler, P. (2007). Glatthard, Karl. Dictionnaire historique de la Suisse. http://www.hls-dhs-dss.ch/ textes/f/F6327.php.

Triet, M. (2001). Attenhofer, Adolf. Dictionnaire historique de la Suisse. http://www.hls-dhs-dss.ch/ textes/f/F13966.php.

von Planta, A. (1978). 50 Jahre Corvigliabahn St. Moritz. Saint-Moritz : Hugo Wetzel.

von Planta, A. (1986). 50 Jahre Skilift Suvretta-Randolins St. Moritz. Saint-Moritz, Gammeter.

Vonnard, P. \& Quin, G., (2019). Promouvoir et jouer au football pendant la guerre. La Fédération internationale de Football Association, les forces de l'Axe et la Deuxième Guerre mondiale. Hispania Nova, 17, 306-340.

Z'Graggen, A. \& Comte, M. (2013). Suvretta House St. Moritz. Zurich : NZZ Libro.

\section{Resumen : Esquiar ... iun deporte "alpino" dirigido por suizos?}

La figura de Marc Hodler se cierne sobre la Federación Internacional de Esquí y sobre el esquí internacional. Con su mandato de 47 años como jefe de la institución (1951-1998), el líder bernés es sin lugar a dudas el líder deportivo con más años en una posición importante en el deporte internacional, pero lejos de ser un hombre aislado, esta trayectoria representa la importancia de las redes de esquí suizas, que reúne a deportistas de turismo deportivo, campeonas y campeones, organizadores de competencias, políticos activos en diferentes niveles del sistema suizo. Como parte de esta contribución, nuestra ambición es doble, tanto para entender las lógicas suizas e internacionales de la construcción de una gobernanza del esquí alrededor de la Segunda Guerra Mundial, como también, más cualitativamente, para medir el influencia de los líderes suizos en los cambios de las diferentes condiciones de esquí (alpino, nórdico) al mismo tiempo. Por lo tanto, será importante para nosotros resaltar la dinámica cruzada que puede existir entre las dos principales regiones claves del esquí en Suiza (Lutschine y Grisons), pero también para profundizar nuestra comprensión de la organización de las redes hervéticas en torno al esquí internacional. Para producir estos análisis, nos basaremos en los archivos institucionales de la FIS, pero también en los documentos 
consultados con la Federación Suiza de Esquí, el Museo Suizo del Deporte, el municipio de St. Moritz y el Comité Olímpico Internacional.

Palabras clave: Esquí, Suiza, Dirigentes, Redes, Alpes

\section{Zusammenfassung: Skifahren.... ein "alpiner" Sport, der von Schweizern geleitet wird?}

Marc Hodlers Figur schwebt über dem Internationalen Skiverband und dem internationalen Skisport. Mit seiner 47-jährigen Amtszeit an der Spitze der Institution (1951-1998) ist der Berner Funktionär zweifellos der Sportführer, der am längsten in einer wichtigen Position im internationalen Sport verblieben ist. Aber Hodler war weit davon entfernt, ein isolierter Mann zu sein, denn sein Werdegang verkörpert die Dichte der Schweizer Skinetzwerke, die Akteure des SportTourismus, Champions, Wettbewerbsveranstalter und Politiker zusammenbringen, die auf den verschiedenen Ebenen des Schweizer Systems tätig sind. Im Rahmen dieses Beitrags verfolgen wir zwei Ziele: einerseits das Verständnis der schweizerischen und internationalen Logik des Aufbaus der Skiverwaltung um die Zeit des Zweiten Weltkriegs, andererseits aber auch die qualitative Beurteilung des Einflusses von Schweizer Führungskräften auf die Veränderungen der verschiedenen Skimodalitäten (alpin, nordisch) in diesem Zeitraum. Es wird uns daher darum gehen, die Querverbindungen zwischen den beiden wichtigsten Skiregionen der Schweiz (Grindelwald und Graubünden) hervorzuheben, aber auch unser Verständnis für die Organisation der Schweizer Netzwerke rund um das internationale Skifahren zu vertiefen. Für diese Analysen stützen wir uns auf das Verbandsarchiv der FIS, aber auch auf Dokumente des Schweizerischen Skiverbandes, des Schweizerischen Sportmuseums, der Gemeinde St. Moritz und des Internationalen Olympischen Komitees.

SCHLaGWÖRTER: Ski, Schweiz, Sportführer, Network, Alpen

\section{Riassunto : Lo sci... uno sport «alpino» diretto da Svizzeri ?}

La figura di Marc Hodler incombe sulla Fédération Internationale de Ski e sullo sci internazionale. Con il suo mandato di 47 anni alla testa dell'istituzione (1951-1998), il dirigente bernese è incontestabilmente il dirigente sportivo rimasto più a lungo in una posizione maggiore in seno allo sport internazionale, ma lontano dall'essere quella di un uomo isolato, questa traiettoria incarna la densità delle reti dello sci svizzero, che raccoglie degli attori del turismo sportivo, delle figure di campionesse e di campioni, degli organizzatori di competizioni e dei politici attivi a differenti livelli del sistema elvetico. Nel quadro di questo contributo, la nostra ambizione è doppia, contemporaneamente di comprendere le logiche svizzere e internazionali della costruzione di una governance dello sci dopo la Seconda Guerra Mondiale, ma anche, in maniera più qualitativa, di misurare l'influenza dei dirigenti elvetici sulle trasformazioni delle differenti modalità di sci (alpino, nordico) in questo stesso periodo. Per questo fatto, per noi si tratterà di sottolineare le dinamiche incrociate che possono esistere tra le due principali regioni chiave dello sci in Svizzera (la Lutchine e i Grigioni), ma anche di approfondire la nostra comprensione dell'organizzazione delle reti elvetiche attorno allo sci internazionale. Per produrre queste analisi ci appoggeremo sugli achivi istituzionali della FIS, ma anche su documenti consultati preso la Fédération Suisse de Ski, del Musée du sport suisse, del comune di St. Moritz e del Comité International Olympique.

Parole chiave : Alpi, dirigente, reti, sci, Svizzera. 